\title{
A characterization of products of balls by their isotropy groups
}

\author{
Axel Hundemer \\ Department of Mathematics, University of Michigan, East Hall, Ann Arbor, MI 48109-1109, \\ USA (e-mail: hundemer@math.lsa.umich.edu)
}

Received: 27 February 1998 / In final form: 6 August 1998

\begin{abstract}
In this paper we will characterize products of balls-especially the ball and the polydisc - in $\mathbb{C}^{n}$ by properties of the isotropy group of a single point. It will be shown that such a characterization is possible in the class of Siegel domains of the second kind, a class that extends the class of bounded homogeneous domains, and that such a characterization is no longer possible in the class of bounded domains with noncompact automorphism groups. The main result is that a Siegel domain of the second kind $G \subset \mathbb{C}^{n}$ is biholomorphically equivalent to a product of balls, iff there is a point $p \in G$ such that the isotropy group of $p$ contains a torus of dimension $n$. As an application it will be proved that the only domains biholomorphically equivalent to a Siegel domain of the second kind and to a Reinhardt domain are exactly the domains biholomorphically equivalent to a product of balls.
\end{abstract}

Mathematics Subject Classification (1991): 32A07, 32M05, 32M15

\section{Notation and definitions}

The unit ball in $\mathbb{C}^{n}$ will be denoted by $B_{n}$, the unit polydisc by $P_{n}$. In this paper we will give characterizations of products of balls up to biholomorphism. So we can assume without loss of generality that all the balls in the paper are actually unit balls.

Let $G$ be a domain in $\mathbb{C}^{n}$. The automorphism group of $G$ will be denoted by $A u t G$, the isotropy group of a point $p \in G$ by $A u t_{p} G$. As usual, these groups will be equipped with the compact open topology (see [Nar71] for an introduction to automorphism groups). Both groups are topological groups with respect to this topology. If $G$ is bounded, they are even real Lie groups, 
with $A u t_{p} G$ being compact. Let $A u t^{\prime} G$ respectively $A u t_{p}^{\prime} G$ denote the connected components of the identity of the groups above.

The class of all domains in $\mathbb{C}^{n}$ biholomorphically equivalent to a

- bounded symmetric domain will be denoted by $\mathbf{B S D}_{n}$,

- bounded homogeneous domain will be denoted by $\mathbf{B H D}_{n}$,

- Siegel domain of the first kind will be denoted by SD1 ${ }_{n}$,

- Siegel domain of the second kind will be denoted by SD2 ${ }_{n}$,

- bounded domain with a noncompact automorphism group will be denoted by $\mathbf{B N C}_{n}$.

If the dimension of the domain is not of importance, the index will be suppressed. The following inclusions are well known:

\section{SD1}

$$
\mathrm{BSD} \subset \mathrm{BHD} \subset \mathrm{SD2} \subset \mathrm{BNC}
$$

Furthermore, $\mathbf{B S D}_{n} \neq \mathbf{B H D}_{n}$ for every $n>3$ (a well known result of I.I. Pyatetskii-Shapiro), $\mathbf{B H D}_{n} \neq \mathbf{S D 2}{ }_{n}$ for $n>2, \mathbf{S D 1}_{n} \neq \mathbf{S D 2} 2_{n}$ for $n>1$, and $\mathbf{S D 2}{ }_{n} \neq \mathbf{B N C}_{n}$ for all $n$. As an application of the main theorem it will be shown in this paper that there are even topological balls in $\mathbf{B N C}_{n} \backslash \mathbf{S D 2} 2_{n}$ for all $n>1$.

For an introduction to bounded symmetric domains see [Hel78], for an introduction to Siegel domains see for example [Mur72]. For the convenience of the reader we will recapitulate the definition of Siegel domains:

Definition A set $C \subset \mathbb{R}^{n}$ is a cone, if $x \in C \Leftrightarrow \lambda x \in C$ holds for all $\lambda \in \mathbb{R}^{+}$. A cone is called regular, if it is nonempty, open, convex and does not contain an entire line (Note that it follows from convexity that $x, y \in C \Rightarrow x+y \in C$ holds for regular cones). Let from now on $C$ denote a regular cone in $\mathbb{R}^{n}$.

The Siegel domain of the first kind over $C \subset \mathbb{R}^{n}$ is the tube domain $\{z \in$ $\left.\mathbb{C}^{n}: \operatorname{Im} z \in C\right\}$.

A $C$-hermitian form is a mapping $H: \mathbb{C}^{k} \times \mathbb{C}^{k} \rightarrow \mathbb{C}^{n}$ with the following properties:

(i) $H$ is $\mathbb{C}$-linear in the first argument

(ii) $H(z, w)=\overline{H(w, z)}$

(iii) $H(z, z) \in \bar{C}$

(iv) $H(z, z)=0 \Leftrightarrow z=0$

Note that this definition coincides with the usual definition of a hermitian form if $C=\mathbb{R}^{+}$. 
The Siegel domain of the second kind over $C$ with $C$-hermitian form $H$ is now defined to be $\left\{(z, w) \in \mathbb{C}^{n+k}: \operatorname{Im} z-H(w, w) \in C\right\}$. The pair $(n, k)$ is called the type of the Siegel domain. It has been shown in [KMO70] that the type is a biholomorphic invariant; therefore it makes sense to speak of the type of a domain in SD2 as well.

Finally, note that $A u t G$ and $A u t_{p} G$ are real Lie groups even if $G \in$ SD2, since every Siegel domain of the second kind is biholomorphically equivalent to a bounded domain.

\section{Introduction}

In this paper we will study characterizations of the ball and the polydisc, as well as arbitrary products of balls, by the structure of an isotropy group of a single point.

Characterizations of the ball are known for quite a while. B. Wong for example proved in [Won77] that a strongly pseudoconvex domain with $C^{2}$ boundary in $\mathbb{C}^{n}$ is biholomorphically equivalent to a ball, iff its automorphism group is noncompact. This result has been generalized by several authors. The proofs of these results are of a differential geometrical nature and make use of smoothness properties of the boundary of the domain. These methods are thus not suitable for a characterization of the polydisc. Instead we will use the Lie group structure of automorphism and isotropy groups.

The starting point is the geometrical observation that the high amount of symmetry of the ball and the polydisc is reflected in the size of the isotropy groups of the origin (for proofs see [Nar71]):

$$
\begin{aligned}
A u t_{0} B_{n}= & \{z \mapsto \mathbf{A} z: \mathbf{A} \in U(n)\} \\
A u t_{0}^{\prime} P_{n}= & \left\{\operatorname{diag}\left(e^{i \varphi_{1}}, \ldots, e^{i \varphi_{n}}\right) \cdot z: \varphi_{j} \in \mathbb{R} \forall j\right\} \cong\left(S^{1}\right)^{n} \\
A u t_{0} P_{n}= & \left\{z \mapsto \mathbf{P} \cdot \operatorname{diag}\left(e^{i \varphi_{1}}, \ldots, e^{i \varphi_{n}}\right) \cdot z: \varphi_{j} \in \mathbb{R} \forall j\right. \\
& \text { and } \mathbf{P} \text { is a permutation matrix }\}
\end{aligned}
$$

Let $B_{k_{1}} \times \cdots \times B_{k_{m}}$ be a product of balls. A theorem of H. Cartan states that $A_{u t}^{\prime}{ }_{(p 1, p 2)} G_{1} \times G_{2} \cong A u t_{p_{1}}^{\prime} G_{1} \times A u t_{p_{2}}^{\prime} G_{2}$ for bounded domains $G_{1}$ and $G_{2}$. Thus

$$
A u t_{0}^{\prime}\left(B_{k_{1}} \times \cdots \times B_{k_{m}}\right)=U\left(k_{1}\right) \times \cdots \times U\left(k_{m}\right)
$$

In all these cases the isotropy groups contain the $n$-dimensional torus of mappings

$$
\left\{z \mapsto \operatorname{diag}\left(e^{i \varphi_{1}}, \ldots, e^{i \varphi_{n}}\right) \cdot z: \varphi_{j} \in \mathbb{R} \forall j\right\}
$$


Since products of balls are homogeneous, we have that

$$
A u t_{p}^{\prime}\left(B_{k_{1}} \times \ldots \times B_{k_{m}}\right) \cong U\left(k_{1}\right) \times \ldots \times U\left(k_{m}\right) \forall p \in B_{k_{1}} \times \ldots \times B_{k_{m}}
$$

so these groups contain a torus of dimension $n$ as well. Thus $A u t_{p} G$ contains a torus of dimension $n$ for all $p \in G$, if $G$ is biholomorphically equivalent to a product of balls.

We will use this property for our characterization. To be more precise: we will investigate if it is possible to characterize products of balls by the property that at least a single isotropy group contains a torus of dimension $n$.

Since we only have conditions on the structure of a single isotropy group, it is natural to presume that such a characterization will be possible in the class of bounded homogeneous domains at most (the isotropy groups of a homogeneous domain are conjugated and thus isomorphic). Surprisingly, the characterization holds as desired not only in BHD, but in the much larger class SD2, a class of domains that lies between BHD and BNC. Our main result is:

Theorem A domain $G \in \mathbf{S D 2}_{n}$ is biholomorphically equivalent to a product of balls, iff there is a point $p \in G$ such that Aut $t_{p} G$ contains a torus of dimension $n$.

Since we will work in the class SD2, we will give realizations of products of balls as Siegel domains of the second kind. It is straightforward to prove that the map

$$
\begin{aligned}
f: & \mathbb{C}^{k+1} \rightarrow \mathbb{C}^{k+1}: \\
& \left(z, w_{1}, \ldots, w_{k}\right) \mapsto\left(z_{1}, \ldots, z_{k+1}\right):=\left(\frac{z-i}{z+i}, \frac{2 w_{1}}{z+i}, \ldots, \frac{2 w_{k}}{z+i}\right)
\end{aligned}
$$

maps the Siegel domain of the second kind

$$
\left\{\left(z, w_{1}, \ldots, w_{n}\right): \operatorname{Im} z-\left|w_{1}\right|^{2}-\cdots-\left|w_{k}\right|^{2}>0\right\}
$$

biholomorphically onto $B_{k+1}$. More general, $G:=B_{k_{1}+1} \times \cdots \times B_{k_{m}+1} \subset$ $\mathbb{C}^{m+k}$ is biholomorphically equivalent to the following Siegel domain of the second kind of type $(m, k)$ :

$$
\tilde{G}:=\left\{\begin{array}{cr}
\operatorname{Im} z_{1}-\left|w_{1}\right|^{2}-\ldots & -\left|w_{k_{1}}\right|^{2}>0 \\
\operatorname{Im} z_{2}-\left|w_{k_{1}+1}\right|^{2}-\ldots & -\left|w_{k_{1}+k_{2}}\right|^{2}>0 \\
\vdots & \\
\operatorname{Im} z_{m}-\left|w_{k_{1}+\cdots+k_{m-1}+1}\right|^{2}-\ldots & -\left|w_{k}\right|^{2}>0
\end{array}\right\}
$$




\section{Preliminaries}

Before we can prove the main theorem of this paper, we will first have to deal with some special cases and other preliminaries. We begin by exploring some of the consequences of the condition of the main theorem.

Lemma 3.1 Let $G \subset \mathbb{C}^{n}$ be a bounded domain. Assume that there is a $p \in G$ such that Aut ${ }_{p} G$ contains a torus $T$ of dimension $n$. Then $T$ is a maximal torus in $A_{u t} G$.

Proof. Let $H:=\left\{D \psi(p): \psi \in A u t_{p} G\right\}$. A theorem of H. Cartan states that

$$
J: A u t_{p} G \rightarrow H, \psi \mapsto D \psi(p)
$$

is an isomorphism of Lie groups. Therefore, $H$ is a compact subgroup of $\operatorname{GL}(n, \mathbb{C})$. Let $\tilde{T}:=J(T)$. Since the maximal tori in $\operatorname{GL}(n, \mathbb{C})$ are exactly the tori of dimension $n, \tilde{T}$ is a maximal torus in $H$; thus $T$ is a maximal torus in $A u t_{p} G$.

Corollary 3.2 Let $\mathbb{C}^{n} \supset G=G_{1} \times \cdots \times G_{k}, G_{j} \subset \mathbb{C}^{n_{j}}$ be a bounded domain, and let $p=\left(p_{1}, \ldots, p_{k}\right), p_{j} \in \mathbb{C}^{n_{j}}$, be a point in $G$. If Aut ${ }_{p} G$ contains a torus of dimension n, then Aut $p_{j} G_{j}$ contains a torus of dimension $n_{j}$ for all $j$.

Proof. Let $m_{j}$ be the dimension of a maximal torus in $A u t_{p_{j}} G_{j}$. Then $m_{1}+\cdots+m_{k} \geq n$. It follows now from lemma 3.1 that in fact $m_{1}+\cdots+$ $m_{k}=n$, and that furthermore $m_{j}=n_{j}$ for all $j$.

Lemma 3.3 Let $G \subset \mathbb{C}^{n}$ be a bounded domain. Assume that there is a $p \in G$ such that Aut $t_{p}$ contains a torus of dimension n. Then there is an involution in $A u t_{p}^{\prime} G$ with an isolated fixed point in $p$.

Proof. Again we will identify $A u t_{p} G$ with the compact subgroup $H:=$ $\left\{D \psi(p): \psi \in A u t_{p} G\right\}$ of $\operatorname{GL}(n, \mathbb{C})$. It is well known that for every compact subgroup $S \leq \mathrm{GL}(n, \mathbb{C})$ there exists a hermitian product $<$, > on $\mathbb{C}^{n}$ such that $S \leq U(n,<,>)$. Especially it follows that every member of $H$ is diagonalizable with eigenvalues of absolute value 1 . Let $\tilde{T}$ be a torus of dimension $n$ in $H$ and let $\mathbf{M} \in \tilde{T}$ such that $\overline{\left\{\mathbf{M}^{k}\right\}_{k \in \mathbb{N}}}=\tilde{T}$ (the set of all such $\mathbf{M}$ is dense in $\tilde{T}$ ). We can assume after a suitable linear change of coordinates that $\mathbf{M}$ is a diagonal matrix. Consequently, $\tilde{T}$ consists solely of diagonal matrices with diagonal elements of absolute value 1 . Since $\tilde{T}$ is a torus of dimension $n$, it follows that

$$
\tilde{T}=\left\{\operatorname{diag}\left(e^{i \alpha_{1}}, \ldots, e^{i \alpha_{n}}\right): \alpha_{1}, \ldots, \alpha_{n} \in \mathbb{R}\right\}
$$


Especially, $-\mathbf{I} \in \tilde{T}$. There is a $\phi \in A u t_{p}^{\prime} G$ with $D \phi(p)=-\mathbf{I}$, and since $-\mathbf{I}$ is an involution in $\operatorname{GL}(n, \mathbb{C}), \phi$ is an involution in $A u t_{p}^{\prime} G$. It follows from the inverse function theorem that $\phi-i d$ has an isolated zero in $p$. In other words, $\phi$ has an isolated fixed point in $p$.

We now prove some special cases of the main theorem.

Proposition 3.4 A domain $G$ in $\mathbf{B S D}_{n}$ is biholomorphically equivalent to a product of balls, iff there is a point $p \in G$ such that Aut ${ }_{p} G$ contains a torus of dimension $n$.

This proposition is an immediate consequence of the classification of bounded symmetric domains by E. Cartan (see [Hel78]). We include the details for the convenience of the reader.

Proof. We assume first that $G$ is an irreducible bounded symmetric domain. There are 4 classes of these domains with members in infinitely many dimensions - called the classical domains of type I-IV_and 2 exceptional domains in $\mathbb{C}^{16}$ respectively $\mathbb{C}^{27}$. Descriptions of the classical domains can be found in [Hel78], descriptions of the two exceptional domains can be found in [Koe62]. We will include the definitions of the classical domains here, since we will need some of the details.

Type I : domains of this type exist in every dimension. Let $n=p q$ with $p \geq q>0$. Identify $\mathbb{C}^{n}$ with the set of all $p \times q$ matrices with complex entries. Let $\mathbf{Z}$ denote a matrix of this type and let $\mathbf{I}_{q}$ denote the $q \times q$ identity matrix. Then the domain of type I with parameters $p$ and $q$ is

$$
R_{I}:=\left\{\mathbf{Z}: \mathbf{I}_{q}-\mathbf{Z}^{*} \mathbf{Z} \text { is positive definite }\right\}
$$

The isotropy groups are isomorphic to $S(U(p) \times U(q))$.

Type II : a domain of this type exists only in dimensions $n$ of the form $n=p(p+1) / 2, p \in \mathbb{N}$. For such an $n$ we can identify $\mathbb{C}^{n}$ with the set of all symmetric $p \times p$ matrices and the domain of type II is defined as

$$
R_{I I}:=\left\{\mathbf{Z}: \mathbf{I}_{p}-\mathbf{Z} \overline{\mathbf{Z}} \text { is positive definite }\right\}
$$

The isotropy groups are isomorphic to $U(p)$.

Type III : a domain of this type exists only in dimensions $n$ of the form $n=p(p+1) / 2, p \in \mathbb{N}$. For such an $n$ we can identify $\mathbb{C}^{n}$ with the set of all skew-symmetric $(p+1) \times(p+1)$ matrices (a skew-symmetric matrix has zeroes on its diagonal). The domain of type III is defined as

$$
R_{I I I}:=\left\{\mathbf{Z}: \mathbf{I}_{p+1}+\mathbf{Z} \overline{\mathbf{Z}} \text { is positive definite }\right\}
$$

The isotropy groups are isomorphic to $U(p)$. 
Type IV : a domain of this type exists in every dimension $n>2$; it is defined by

$$
R_{I V}:=\left\{z:\left|z z^{t}\right|-2 \bar{z} z^{t}+1>0,\left|z z^{t}\right|<1\right\}
$$

The isotropy groups are isomorphic to $S O(n) \times S O(2)$.

Classical domains of different types may coincide in low dimensions; for example, the domains of type I with $p=q=1$, type II with $p=1$, and type III with $p=1$ are all just the unit disc in $\mathbb{C}$.

Finally, the Lie algebras of the isotropy groups of the exceptional domains in $\mathbb{C}^{16}$ respectively $\mathbb{C}^{27}$ are $\mathfrak{s o}(10)+\mathbb{R}$ respectively $\mathfrak{e}_{6}+\mathbb{R}$.

The isotropy groups of $G$ can thus only contain a torus of dimension $n$ if $G$ is of type I with $q=1$ (or of type II or III with $p=1$, but these cases are redundant by the remark above). But the type I domain with $q=1, p=n$ is just the unit ball in $\mathbb{C}^{n}$.

Now let $G=G_{1} \times \cdots \times G_{k}$ be a bounded symmetric domain with irreducible factors $G_{j}$. It follows immediately from corollary 3.2 and the considerations above that $G$ is biholomorphically equivalent to a product of balls.

Note that it follows immediately from lemma 3.3 that proposition 3.4 holds as well in the larger class BHD. We will not use this result in the rest of the paper, and it will be superseded by the main theorem 4.1.

In conjunction with a theorem by O.S. Rothaus, 3.3 and 3.4 can also be used to characterize the polydisc in SD1:

\section{Proposition 3.5}

(i) A domain $G$ in SD1 is biholomorphically equivalent to a product of balls, iff it is biholomorphically equivalent to a polydisc.

(ii) A domain $G$ in $\mathbf{S D} 1_{n}$ is biholomorphically equivalent to a polydisc, iff there is a point $p \in G$ such that Aut $t_{p} G$ contains a torus of dimension $n$.

(iii) A domain $G$ in $\mathbf{S D} 1_{n}$ is biholomorphically equivalent to a polydisc, iff there is a point $p \in G$ such that Aut ${ }_{p}^{\prime} G$ is a torus of dimension n.

Proof. Let $P_{n}$ be the (unit) polydisc in $\mathbb{C}^{n}$. It has already been mentioned in the introduction that $A u t_{0}^{\prime} P_{n}$ is a torus of dimension $n$ (as is every other group $A u t_{p}^{\prime} P_{n}, p \in P_{n}$ ). The condition of (iii) is thus fulfilled for the polydisc. Furthermore, $P_{n}$ is biholomorphically equivalent to the Siegel domain of the first kind $\left(H^{+}\right)^{n}$, where $H^{+}$is the upper half plane in $\mathbb{C}$.

Let $G$ be a domain in $\mathbf{S D} \mathbf{1}_{n}$, which is biholomorphically equivalent to a product of balls. Then the type of $G$ is $(n, 0)$. It has been shown in the introduction that the type of the product of balls $B_{k_{1}+1} \times \cdots \times B_{k_{m}+1} \subset$ 
$\mathbb{C}^{m+k}, k=k_{1}+\cdots+k_{m}$, is $(m, k)$. Thus $k_{1}=\cdots=k_{m}=0$, and $G$ is biholomorphically equivalent to a polydisc. This proves ((i)).

Now let $G$ be in SD1. A theorem of O.S. Rothaus ([Rot71], introduction respectively theorem 18) states that a Siegel domain of the first kind is already biholomorphically equivalent to a bounded symmetric domain, if there is an involution in $A u t G$ with an isolated fixed point in $G$. It follows from lemma 3.3 and proposition 3.4 that $G$ is biholomorphically equivalent to a product of balls. Now (i) shows that $G$ is biholomorphically equivalent to a polydisc, which proves (ii) and (iii).

\section{A characterization of products of balls in the class SD2}

We will prove our main theorem about a characterization of products of balls in the class of Siegel domains of the second kind in this section. Additionally we will give characterizations of the two most important special cases of products of balls: the ball and the polydisc.

Theorem 4.1 A domain $G \in \mathbf{S D 2}{ }_{n}$ is biholomorphically equivalent to a product of balls, iff there is a point $p \in G$ such that Aut ${ }_{p} G$ contains a torus of dimension $n$.

Proof. Let $n=m+k, z \in \mathbb{C}^{m}, w \in \mathbb{C}^{k}$ and $G:=\left\{(z, w) \in \mathbb{C}^{m+k}\right.$ : $\operatorname{Im} z-H(w, w) \in C\}$ with a regular cone $C \subseteq \mathbb{R}^{m}$ and a $C$-hermitian form $H: \mathbb{C}^{k} \times \mathbb{C}^{k} \rightarrow \mathbb{C}^{m}$. Since the theorem has already been proved in SD1, we can assume $k>0$. In several steps we will use information about Aut $G$ to receive information about $G$ and vice versa.

Step 1: Let $p=(u, v), u \in \mathbb{C}^{m}, v \in \mathbb{C}^{k}$. It is well known that the affine mappings

$$
\Phi_{a, b}:\left(\begin{array}{c}
z \\
w
\end{array}\right) \mapsto\left(\begin{array}{c}
z+a+2 i H(w, b)+i H(b, b) \\
w+b
\end{array}\right), a \in \mathbb{R}^{m}, b \in \mathbb{C}^{k}
$$

are automorphisms of $G$ (see [Mur72]). Obviously $\Phi_{0,-v}(u, v)=(\tilde{u}, 0)$ with a $\tilde{u} \in \mathbb{C}^{m}$. Therefore $A u t_{(u, v)}^{\prime} G$ and $A u t_{(\tilde{u}, 0)}^{\prime} G$ are isomorphic. We will assume without loss of generality that $p=(u, 0)$.

Let $U$ be a torus of dimension $n$ in $A u t_{p}^{\prime} G$. It follows from lemma 3.1 that $U$ is a maximal torus in the compact and connected Lie group $A u t_{p}^{\prime} G$. A well known theorem in Lie group theory states that all maximal tori in a compact and connected Lie group are conjugated by inner automorphisms, and that every torus in the Lie group is contained in a maximal torus.

Since $p=(u, 0)$, the mappings $\varphi(z, w):=\left(z, e^{i \psi} w\right), \psi \in \mathbb{R}$, form a one dimensional torus in $A u t_{p}^{\prime} G$. There is a maximal torus $U_{\varphi} \subseteq A u t_{p}^{\prime} G$ 
that contains all these mappings. Since $U$ and $U_{\varphi}$ are conjugated, we may assume $U=U_{\varphi}$.

Step 2: Let $F(z, w):=(f(z, w), g(z, w))$, with

$$
f=\left(f_{1}, \ldots, f_{m}\right), g=\left(g_{1}, \ldots, g_{k}\right)
$$

be an arbitrary automorphism in $U$, and let $\varphi(z, w):=\left(z, e^{i \psi} w\right)$. Since $U$ is abelian, we have

$$
\begin{aligned}
& F \circ \varphi(z, w)=\left(f\left(z, e^{i \psi} w\right), g\left(z, e^{i \psi} w\right)\right)= \\
& \varphi \circ F(z, w)=\left(f(z, w), e^{i \psi} g(z, w)\right) \\
& \Leftrightarrow f(z, w)=f\left(z, e^{i \psi} w\right) \wedge e^{i \psi} g(z, w)=g\left(z, e^{i \psi} w\right)
\end{aligned}
$$

for all $(z, w) \in G$ and all $\psi \in \mathbb{R}$.

Let the power series expansion of $F$ at $0 \in \mathbb{C}^{k}$ with fixed $z$ be

$$
f_{j}(z, w)=\sum_{\nu \in \mathbb{N}_{0}^{k}} a_{\nu}^{j}(z) w^{\nu}, g_{j}(z, w)=\sum_{\nu \in \mathbb{N}_{0}^{k}} b_{\nu}^{j}(z) w^{\nu}
$$

Therefore

$$
\begin{aligned}
f_{j}(z, w) & =\sum_{\nu \in \mathbb{N}_{0}^{k}} a_{\nu}^{j}(z) w_{1}^{\nu_{1}} \cdot \ldots \cdot w_{k}^{\nu_{k}} \\
& =\sum_{\nu \in \mathbb{N}_{0}^{k}} a_{\nu}^{j}(z)\left(e^{i \psi \nu_{1}} \cdot w_{1}^{\nu_{1}}\right) \cdot \ldots \cdot\left(e^{i \psi \nu_{1}} \cdot w_{k}^{\nu_{k}}\right) \\
& =\sum_{\nu \in \mathbb{N}_{0}^{k}} a_{\nu}^{j}(z) e^{i \psi \cdot|\nu|} w^{\nu}
\end{aligned}
$$

and

$$
\begin{aligned}
e^{i \psi} g_{j}(z, w) & =e^{i \psi} \sum_{\nu \in \mathbb{N}_{0}^{k}} b_{\nu}^{j}(z) w_{1}^{\nu_{1}} \cdot \ldots \cdot w_{k}^{\nu_{k}} \\
& =\sum_{\nu \in \mathbb{N}_{0}^{k}} b_{\nu}^{j}(z)\left(e^{i \psi \nu_{1}} \cdot w_{1}^{\nu_{1}}\right) \cdot \ldots \cdot\left(e^{i \psi \nu_{1}} \cdot w_{k}^{\nu_{k}}\right) \\
& =\sum_{\nu \in \mathbb{N}_{0}^{k}} b_{\nu}^{j}(z) e^{i \psi \cdot|\nu|} w^{\nu}
\end{aligned}
$$

Comparing the coefficients yields $a_{\nu}^{j}(z)=0$, if $|\nu|>0$, and $b_{\nu}^{j}(z)=$ 0 , if $|\nu| \neq 1$. It follows that $f$ is independent of $w$, and that $g$ is of the form $g(z, w):=\mathbf{A}(z) \cdot w$, where $\mathbf{A}(z)$ is a matrix whose components are holomorphic functions in $z$. The domain of definition of $f$ and $\mathbf{A}$ is 
the set $\tilde{G}:=\left\{z \in \mathbb{C}^{m}: \exists w \in \mathbb{C}^{k}\right.$ with $\left.\operatorname{Im} z-H(w, w) \in C\right\}$. Since $H(w, w) \in \bar{C}$, and since $C$ is an open convex cone we have

$$
\operatorname{Im} z-H(w, w) \in C \Rightarrow(\operatorname{Im} z-H(w, w))+H(w, w) \in C
$$

Consequently $\tilde{G}=\left\{z \in \mathbb{C}^{m}: \operatorname{Im} z \in C\right\}=\left\{z \in \mathbb{C}^{m}:(z, 0) \in G\right\}$. Thus $\tilde{G}$ is a Siegel domain of the first kind in $\mathbb{C}^{m}$.

Summarizing, we have that every automorphism in $U$ is of the form $F(z, w)=(f(z), \mathbf{A}(z) \cdot w)$ with holomorphic maps $f: \tilde{G} \rightarrow \mathbb{C}^{m}$ and $\mathbf{A}: \tilde{G} \rightarrow \operatorname{Mat}(k \times k, \mathbb{C})$. If we want to emphasize that these maps depend on $F$, we will denote them by $f_{F}$ respectively $\mathbf{A}_{F}$.

Step 3: Let $F$ be arbitrary in $U$. We will prove that $\mathbf{A}(z)$ is invertible for all $z \in \tilde{G}$, and that $f$ is an automorphism of $\tilde{G}$ that fixes $u$.

$$
\begin{aligned}
D F(z, w) & =\left(\begin{array}{cc}
D f(z) & 0 \\
* & \mathbf{A}(z)
\end{array}\right) \Rightarrow \\
\operatorname{det} D F(z, w) & =\operatorname{det} D f(z) \cdot \operatorname{det} \mathbf{A}(z) \neq 0
\end{aligned}
$$

Thus $\mathbf{A}$ is invertible.

$z \in \tilde{G} \Rightarrow F(z, 0)=(f(z, 0), 0) \in G \Rightarrow f(z) \in \tilde{G} \Rightarrow f(\tilde{G}) \subseteq \tilde{G}$.

$f(z)=f\left(z^{\prime}\right) \Rightarrow F(z, 0)=(f(z), 0)=\left(f\left(z^{\prime}\right), 0\right)=F\left(z^{\prime}, 0\right) \Rightarrow z=z^{\prime}$. Thus $f$ is injective.

$z^{\prime} \in \tilde{G} \Rightarrow\left(z^{\prime}, 0\right) \in G \Rightarrow$ there is $(z, w) \in G$ with $F(z, w)=(f(z), \mathbf{A}(z)$. $w)=\left(z^{\prime}, 0\right)$. Since $\mathbf{A}(z)$ is invertible, it follows that $w=0$. Thus $z \in \tilde{G}$ and $f(z)=z^{\prime}$. Furthermore $(u, 0)=F(u, 0)=(f(u), 0) \Rightarrow f(u)=u$, and it follows that $f \in A u t_{u} \tilde{G}$. Obviously we can even conclude that $f \in A u t_{u}^{\prime} \tilde{G}$.

Step 4: We will prove in this step that

$$
\tilde{U}:=\{f: \exists F \in U \text { such that } F(z, w)=(f(z), \mathbf{A}(z) \cdot w)\} \subseteq A u t_{u}^{\prime} \tilde{G}
$$

is a torus of dimension $m$. We will use this fact to simplify the shape of $G$ by a linear change of coordinates.

The $n$-dimensional torus $U$ can be identified as a Lie group with $T:=$ $\{D F(p): F \in U\}$ according to a theorem of H. Cartan. A calculation shows

$$
\begin{gathered}
D F(z, w)=\left(\begin{array}{cc}
D f(z) & 0 \\
\mathbf{B}(z, w) \mathbf{A}(z)
\end{array}\right) \text { with } \\
\mathbf{B}_{i j}(z, w)=\frac{\partial a_{i 1}}{\partial z_{j}}(z) \cdot w_{1}+\cdots+\frac{\partial a_{i k}}{\partial z_{j}}(z) \cdot w_{k}
\end{gathered}
$$


where $\mathbf{A}(z)=\left(a_{i j}(z)\right)$. Consequently,

$$
D F(p)=D F(u, 0)=\left(\begin{array}{cc}
D f(u) & 0 \\
0 & \mathbf{A}(u)
\end{array}\right)
$$

Define

$$
\begin{aligned}
& T_{1}:=\{D f(u): f \in \tilde{U}\} \text { and } \\
& T_{2}:=\{\mathbf{A}(u): \exists F \in U \text { with } F(z, w)=(f(z), \mathbf{A}(z) \cdot w), \\
& f \in \tilde{U} \text { arbitrary }\}
\end{aligned}
$$

The projections $p_{1}: T \rightarrow T_{1}, D F(p) \mapsto D f(u)$ and $p_{2}: T \rightarrow T_{2}$, $D F(p) \mapsto \mathbf{A}(u)$ are morphisms of Lie groups, therefore $T_{1} \subseteq \operatorname{GL}(m, \mathbb{C})$ and $T_{2} \subseteq \mathrm{GL}(k, \mathbb{C})$ are tori. We have $n=m+k=\operatorname{dim} T \leq \operatorname{dim} T_{1}+$ $\operatorname{dim} T_{2} \leq m+k$. Thus $\operatorname{dim} T_{1}=m, \operatorname{dim} T_{2}=k$, and $T \cong T_{1} \times T_{2}$. Since $\tilde{U} \cong T_{1}$, we have that $\tilde{U}$ is a torus of dimension $m$. Furthermore we have for all $f \in \tilde{U}$ that the set $T_{2}^{f}:=\{\mathbf{A}(u): \exists F \in U$ with $F(z, w)=$ $(f(z), \mathbf{A}(z) \cdot w)\}=T_{2} \cong\left\{D F(p): F \in U\right.$ and $\left.f_{F}=f\right\} \cong\{F \in U:$ $\left.f_{F}=f\right\}$ is a torus of dimension $k$.

We will use this to simplify the shape of $G$. We just showed that $A u t_{u} \tilde{G}$ contains a torus of dimension $m$, where $\tilde{G}$ is a Siegel domain of the first kind in $\mathbb{C}^{m}$. It follows from theorem 3.5 that $\tilde{G}$ is biholomorphically equivalent to a polydisc, and thus to the Siegel domain of the first kind $T\left(\left(\mathbb{R}^{+}\right)^{m}\right)$ over the cone $\left(\mathbb{R}^{+}\right)^{m}$. It follows from a theorem by Kaup, Matsushima and Ochiai ([KMO70]) that $\tilde{G}$ is linearly equivalent to $T\left(\left(\mathbb{R}_{+}\right)^{m}\right)$, and thus that $G$ is linearly equivalent to a Siegel domain of the second kind over the cone $\left(\mathbb{R}^{+}\right)^{m}$. We may assume without loss of generality that $\left(\mathbb{R}^{+}\right)^{m}$ is the cone of $G$.

Then $\tilde{G}=T\left(\left(\mathbb{R}_{+}\right)^{m}\right)=\left(H^{+}\right)^{m}$, and $f \in A u t_{u}^{\prime}\left(H^{+}\right)^{m}$, where $H^{+}$denotes the upper half plane in $\mathbb{C}$. A theorem of $\mathrm{H}$. Cartan states that $A u t_{\left(p_{1}, p_{2}\right)}^{\prime} G_{1} \times G_{2} \cong A u t_{p_{1}}^{\prime} G_{1} \times A u t_{p_{2}}^{\prime} G_{2}$ for bounded domains $G_{1}, G_{2}$. Thus $f(z)=\left(f_{1}\left(z_{1}\right), \ldots, f_{m}\left(z_{m}\right)\right)$ with $f_{j} \in A u t_{u_{j}}^{\prime} H^{+}, u=$ $\left(u_{1}, \ldots, u_{m}\right)$ and $z=\left(z_{1}, \ldots, z_{m}\right)$. Consequently,

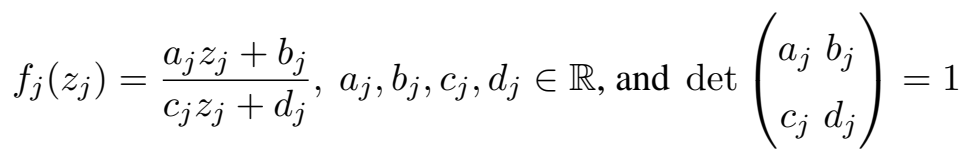

It follows from the definition that the $\left(\mathbb{R}^{+}\right)^{m}$-hermitian form $H$ is of the form $H=\left(\mathbf{H}_{1}, \ldots, \mathbf{H}_{m}\right)$, with positive semi-definite hermitian $k \times k$-matrices $\mathbf{H}_{1}, \ldots, \mathbf{H}_{m}$. 
Step 5: Let $z_{0}=\left(z_{0}^{1}, \ldots, z_{0}^{m}\right) \in \tilde{G}$ be arbitrary. Define

$$
\begin{aligned}
G\left(z_{0}\right):= & \left\{w \in \mathbb{C}^{k}:\left(z_{0}, w\right) \in G\right\} \\
= & \left\{\begin{array}{cc} 
& \operatorname{Im} z_{0}^{1}-w^{*} \mathbf{H}_{1} w>0 \\
w \in \mathbb{C}^{k}: & \vdots \\
& \operatorname{Im} z_{0}^{m}-w^{*} \mathbf{H}_{m} w>0
\end{array}\right\}
\end{aligned}
$$

Obviously, $G\left(z_{0}\right)$ is a bounded circular domain that contains the origin. It will be shown in this step that $G\left(z_{0}\right)$ is even linearly equivalent to a Reinhardt domain.

Let $U_{1}:=\left\{F \in U: f_{F}=i d\right\}=\{F \in U: F(z, w)=(z, \mathbf{A}(z) \cdot w)\}$. It has been shown in the fourth step that $U_{1}$ is a torus of dimension $k$. Every $F \in U_{1}$ fixes every point of the form $\left(z_{0}, 0\right)$ with $z_{0} \in \tilde{G}$. We have $U_{1} \cong\left\{D F\left(z_{0}, 0\right): F \in U_{1}\right\} \cong\left\{\mathbf{A}_{F}\left(z_{0}\right): F \in U_{1}\right\}$ for all $z_{0} \in \tilde{G}$. Define for all $F \in U_{1}$ the mapping

$$
F_{z_{0}}: G\left(z_{0}\right) \rightarrow G\left(z_{0}\right), w \mapsto F\left(z_{0}, w\right)
$$

and define $U\left(z_{0}\right):=\left\{F_{z_{0}}: F \in U_{1}\right\}$. Then $U\left(z_{0}\right)$ is a Lie group of automorphisms of $G\left(z_{0}\right)$ for all $z_{0} \in \tilde{G}$, and $U_{1} \cong U\left(z_{0}\right)$.

This means that $U\left(z_{0}\right)$ is for all $z_{0} \in \tilde{G}$ a $k$-dimensional torus of automorphisms of the bounded circular domain $G\left(z_{0}\right) \ni 0$ fixing the origin. Consequently, $U\left(z_{0}\right)$ consists solely of linear maps. It follows now like in the proof of lemma 3.3 that

$$
U\left(z_{0}\right)=\left\{\operatorname{diag}\left(e^{i \alpha_{1}}, \ldots, e^{i \alpha_{k}}\right): \alpha_{1}, \ldots, \alpha_{n} \in \mathbb{R}\right\}
$$

up to a linear change of coordinates. $U\left(z_{0}\right)$ is thus linearly equivalent to a bounded Reinhardt domain that contains the origin.

Step 6: We will show in this step that the matrices $\mathbf{H}_{j}$ are simultaneously diagonalizable. It will be concluded that after a suitable linear change of coordinates in $w$ every $\mathbf{H}_{j}$ is a diagonal matrix.

We will begin by proving that each two of the matrices $\mathbf{H}_{j}$ are simultaneously diagonalizable. Let $p, q$ with $1 \leq p, q \leq m$, be arbitrary. If $\mathbf{H}_{p}=\mathbf{H}_{q}$, there is nothing to prove. Assume therefore $\mathbf{H}_{p} \neq \mathbf{H}_{q}$. We will distinguish two cases.

1. Case: $\mathbf{H}_{j} \neq \mathbf{0}$ for all $j$.

In this case $W_{j}:=\left\{w \in \mathbb{C}^{k}: w^{*} \mathbf{H}_{j} w=0\right\}$ is a subspace of $\mathbb{C}^{k}$ with a codimension of at least 1 for all $j . W:=\bigcup_{j=1}^{m} W_{j}$ is thus a closed set of Lebesgue measure zero in $\mathbb{C}^{k}$. Let $w_{0} \in \mathbb{C}^{k} \backslash W$ arbitrary, and let

$$
z_{0}^{p}:=i w_{0}^{*} \mathbf{H}_{p} w_{0}, z_{0}^{q}:=i w_{0}^{*} \mathbf{H}_{q} w_{0}, \operatorname{Im} z_{0}^{j}>w_{0}^{*} \mathbf{H}_{j} w_{0} \forall j \neq p, q
$$


With $z_{0}:=\left(z_{0}^{1}, \ldots, z_{0}^{m}\right)$ we have $z_{0} \in \tilde{G}=T\left(\mathbb{R}_{+}^{m}\right)$, and

$$
\operatorname{Im} z_{0}^{j}-w_{0}^{*} \mathbf{H}_{j} w_{0} \begin{cases}>0 & \text { for } j \neq p, q \\ =0 & \text { for } j=p, q\end{cases}
$$

Thus $\left(z_{0}, w_{0}\right) \in \partial G$ and $w_{0} \in \partial G\left(z_{0}\right)$. With $G_{j}\left(z_{0}\right):=\left\{w \in \mathbb{C}^{k}\right.$ : $\left.\operatorname{Im} z_{j}^{0}-w^{*} \mathbf{H}_{j} w>0\right\}$ we have $G\left(z_{0}\right)=\bigcap_{j=1}^{m} G_{j}\left(z_{0}\right)$. By the definition of $z_{0}$ it follows that in a neighborhood $V$ of $w_{0}$ every boundary point of $G\left(z_{0}\right)$ is in in the boundary of $G_{p}\left(z_{0}\right)$ or $G_{q}\left(z_{0}\right)$. Since $\mathbf{H}_{p} \neq \mathbf{H}_{q}$, we can choose $w_{0} \in \mathbb{C}^{k} \backslash W$ in such a way that $G_{p}\left(z_{0}\right)$ and $G_{q}\left(z_{0}\right)$ intersect transversally in $w_{0}$. Thus there are boundary points of $G_{p}\left(z_{0}\right)$ as well as boundary points of $G_{q}\left(z_{0}\right)$ in $V$, which are boundary points of $G\left(z_{0}\right)$. It has been shown in the 5th step that $G\left(z_{0}\right)$ is linearly equivalent to a bounded Reinhardt domain that contains the origin. We may assume after a linear change of coordinates that $G\left(z_{0}\right)$ is such a domain. It will now be proved that both $G_{p}\left(z_{0}\right)$ and $G_{q}\left(z_{0}\right)$ are Reinhardt domains.

Claim $G_{p}\left(z_{0}\right)$ and $G_{q}\left(z_{0}\right)$ are bounded Reinhardt domains containing the origin. Both $\mathbf{H}_{p}$ and $\mathbf{H}_{q}$ are diagonal matrices.

Proof. As has been shown above, there are points $v_{p}, v_{q} \in V$ and neighborhoods $V_{p}, V_{q}$ of $v_{p}$ respectively $v_{q}$ in $V$, such that all boundary points of $G\left(z_{0}\right)$ in $V_{p}$ are boundary points of $G_{p}\left(z_{0}\right)$, and that all boundary points of $G\left(z_{0}\right)$ in $V_{q}$ are boundary points of $G_{q}\left(z_{0}\right)$. Since $G\left(z_{0}\right)$ is a Reinhardt domain, there is for each $w \in \partial G_{j}\left(z_{0}\right) \cap V_{j}, j \in\{p, q\}$, an $\epsilon>0$ such that even $\left(e^{i \varphi_{1}} w_{1}, \ldots, e^{i \varphi_{k}} w_{k}\right) \in \partial G_{j}\left(z_{0}\right)$ for all $\left.\varphi_{1}, \ldots, \varphi_{k} \in\right]-\epsilon, \epsilon[$. Thus, the function

$$
\left(\varphi_{1}, \ldots, \varphi_{k}\right) \mapsto\left(e^{-i \varphi_{1}} \bar{w}_{1}, \ldots, e^{-i \varphi_{k}} \bar{w}_{k}\right) \mathbf{H}_{j}\left(\begin{array}{c}
e^{i \varphi_{1}} w_{1} \\
\vdots \\
e^{i \varphi_{k}} w_{k}
\end{array}\right)
$$

is constantly equal to $\operatorname{Im} z_{0}^{j}$ on $]-\epsilon, \epsilon$. Expanding yields at once that all the coefficients of $\mathbf{H}_{j}$, which are not on the main diagonal, have to be zero. Consequently, $\mathbf{H}_{j}$ is a diagonal matrix, and $G_{j}\left(z_{0}\right)$ is a bounded Reinhardt domain containing the origin for $j \in\{p, q\}$.

Summarizing, we have shown that the matrices $\mathbf{H}_{p}$ and $\mathbf{H}_{q}$ are simultaneously diagonalizable for arbitrary $1 \leq p, q \leq m$. Thus all the matrices $\mathbf{H}_{j}$ are simultaneously diagonalizable. We may assume after a linear change of coordinates in $w$ that every $\mathbf{H}_{j}$ is diagonal. Let $\mathbf{H}_{j}=\operatorname{diag}\left(h_{j 1}, \ldots, h_{1 k}\right)$. Then $G$ has the form 


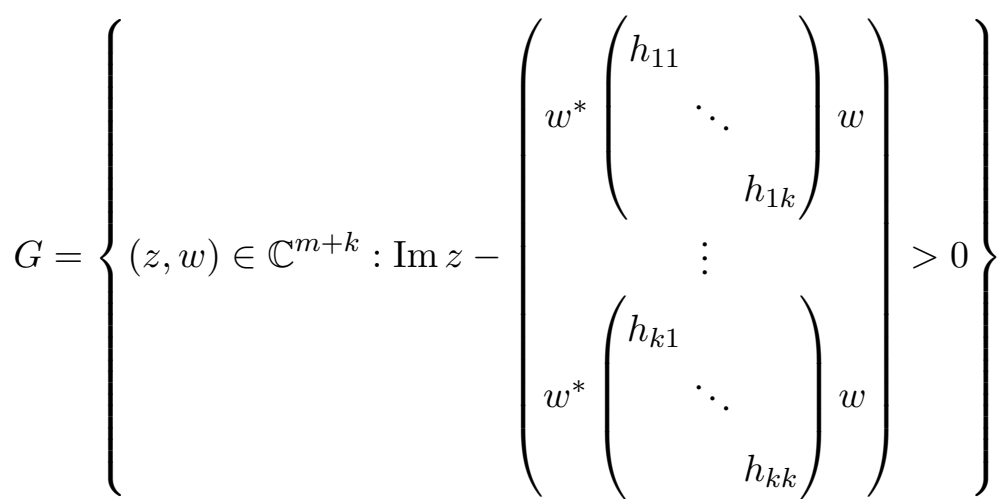

$$
\begin{aligned}
& =\left\{(z, w) \in \mathbb{C}^{m+k}: \operatorname{Im} z-\left(\begin{array}{ccc}
h_{11} & \ldots & h_{1 k} \\
\vdots & & \vdots \\
h_{k 1} & \ldots & h_{k k}
\end{array}\right)\left(\begin{array}{c}
\left|w_{1}\right|^{2} \\
\vdots \\
\left|w_{k}\right|^{2}
\end{array}\right)>0\right\}
\end{aligned}
$$

Define $H:=\left(\begin{array}{ccc}h_{11} & \ldots & h_{1 k} \\ \vdots & & \vdots \\ h_{k 1} & \ldots & h_{k k}\end{array}\right)$, and $H|w|^{2}:=H\left(\begin{array}{c}\left|w_{1}\right|^{2} \\ \vdots \\ \left|w_{k}\right|^{2}\end{array}\right)$. Then $G$ takes the form

$$
G=\left\{(z, w) \in \mathbb{C}^{m+k}: \operatorname{Im} z-H|w|^{2}>0\right\}
$$

Since the matrices $\mathbf{H}_{j}$ are positive semi-definite, the coefficients of $H$ are non-negative real numbers.

2. Case: $\mathbf{H}_{j}=\mathbf{0}$ for at least one $j$.

Assume $\mathbf{H}_{1}, \ldots \mathbf{H}_{r} \neq \mathbf{0}$, and $\mathbf{H}_{r+1}, \ldots, \mathbf{H}_{m}=\mathbf{0}$. In this case, $G$ is the product of the Siegel domain of the second kind

$$
G^{\prime}:=\left\{\left(z_{1}, \ldots, z_{r}, w\right) \in \mathbb{C}^{r+k}: \begin{array}{c}
\operatorname{Im} z_{1}-w^{*} \mathbf{H}_{1} w>0 \\
\\
\\
\operatorname{Im} z_{r}-w^{*} \mathbf{H}_{r} w>0
\end{array}\right\}
$$

and the Siegel domain of the first kind $\left(H^{+}\right)^{m-r}$. It has been shown in the first case that the matrices $\mathbf{H}_{1}, \ldots, \mathbf{H}_{r}$, and thus of course the matrices $\mathbf{H}_{1}, \ldots, \mathbf{H}_{m}$ are simultaneously diagonalizable. Therefore $G$ takes the same simplified form as in the first case, after a linear change of coordinates in $w$.

Step 7: We will use the simplified shape of $G$ to gain further information about the automorphisms $F \in U$.

$G=\left\{(z, w) \in \mathbb{C}^{n}: \operatorname{Im} z>H|w|^{2}\right\} \Rightarrow(z, w) \mapsto\left(z, e^{i \psi_{1}} w_{1}, \ldots, e^{i \psi_{k}} w_{k}\right)$ 
is an automorphism of $G$ for arbitrary $\psi_{1}, \ldots, \psi_{k} \in \mathbb{R}$. The set of all these mappings forms a torus $\tilde{U}$ of dimension $k$. There is a maximal torus in $A u t_{p}^{\prime} G$ that contains $\tilde{U}$. Since all the maximal tori in $A u t_{p}^{\prime} G$ are conjugated, we can assume without loss of generality that $\tilde{U} \subset U$. Let $F \in U, F(z, w)=$ $(f(z), \mathbf{A}(z) \cdot w)$ and $\varphi(z, w):=\left(z, e^{i \psi_{1}} w_{1}, \ldots, e^{i \psi_{k}} w_{k}\right)$ with arbitrary $\psi_{1}, \ldots, \psi_{k} \in \mathbb{R}$. Since $U$ is abelian, we have $F \circ \varphi=\varphi \circ F$. Consequently,

$$
\left(f(z), \mathbf{A}(z)\left(\begin{array}{ccc}
e^{i \psi_{1}} & & \\
& \ddots & \\
& & e^{i \psi_{k}}
\end{array}\right) \cdot w\right)=\left(f(z),\left(\begin{array}{ccc}
e^{i \psi_{1}} & & \\
& \ddots & \\
& & e^{i \psi_{k}}
\end{array}\right) \mathbf{A}(z) \cdot w\right)
$$

With $\mathbf{A}(z):=\left(a_{j l}(z)\right)_{\substack{1 \leq j \leq k \\ 1 \leq l \leq k}}$, this is equivalent to

$$
a_{j l}(z) e^{i \psi_{l}}=e^{i \psi_{j}} a_{j l}(z) \forall j, l
$$

Thus $a_{j l}(z) \equiv 0$ for $j \neq l$. It follows that the matrix $\mathbf{A}_{F}(z)$ is diagonal for all $F \in U$ and all $z \in \tilde{G}$. Summarizing, we have that every $F \in U$ is of the form

$$
F(z, w)=\left(f_{1}\left(z_{1}\right), \ldots, f_{m}\left(z_{m}\right), a_{1}(z) w_{1}, \ldots, a_{k}(z) w_{k}\right)
$$

with $f_{j} \in A u t_{u_{j}}^{\prime} H^{+}$.

Step 8: It has been shown in the 6th step that the components of the matrix $H=\left(h_{j l}\right)_{\substack{1 \leq j \leq m \\ 1<l<k}}$ are non-negative real numbers. It will be shown additionally in this step that exactly one element in each column of $H$ is different from 0 .

Assume without loss of generality that in the $l$-th column the elements $h_{1 l}, \ldots, h_{r l}$ are positive, and the elements $h_{r+1, l}, \ldots, h_{m l}$ are zero.

Let $z_{0}:=i \cdot\left(h_{1 l}, \ldots, h_{r l}, 1, \ldots, 1\right)$, let $w_{0}$ denote the $l$-th member of the standard basis of $\mathbb{C}^{k}$, and let $\lambda \in \mathbb{R}^{+}$be arbitrary.

$$
\begin{aligned}
&(z, w) \in G \Longleftrightarrow \operatorname{Im} z_{1}-h_{11}\left|w_{1}\right|^{2}-\cdots-h_{1 k}\left|w_{k}\right|^{2}>0 \\
& \vdots \\
& \operatorname{Im} z_{m}-h_{m 1}\left|w_{1}\right|^{2}-\cdots-h_{m k}\left|w_{k}\right|^{2}>0
\end{aligned}
$$

For $\left(\lambda^{2} z_{0}, \lambda w_{0}\right)$ we have:

$$
\begin{aligned}
& \operatorname{Im} \lambda^{2} i h_{1 l}-h_{1 l}|\lambda|^{2}=0 \\
& \vdots \\
& \operatorname{Im} \lambda^{2} i h_{r l}-h_{r l}|\lambda|^{2}=0 \\
& \operatorname{Im} \lambda^{2} i-h_{r+1, l}|\lambda|^{2}=\lambda^{2}>0 \\
& \vdots \\
& \operatorname{Im} \lambda^{2} i-h_{m l}|\lambda|^{2}=\lambda^{2}>0
\end{aligned}
$$


$\Rightarrow\left(\lambda^{2} z_{0}, \lambda w_{0}\right) \in \partial G$. Since every $F \in U$ is defined on $T\left(\mathbb{R}_{+}^{m}\right) \times \mathbb{C}^{k} \ni$ $\left(\lambda^{2} z_{0}, \lambda w_{0}\right)$, we have $F\left(\lambda^{2} z_{0}, \lambda w_{0}\right) \in \partial G \forall F \in U$. Let

$$
F(z, w)=\left(f_{1}\left(z_{1}\right), \ldots, f_{m}\left(z_{m}\right), a_{1}(z) w_{1}, \ldots, a_{k}(z) w_{k}\right)
$$

with $f_{j} \in A u t_{u_{j}} H^{+}$. Then

$$
\begin{aligned}
F\left(\lambda^{2} z_{0}, \lambda w_{0}\right)= & \left(f_{1}\left(\lambda^{2} i h_{1 l}\right), \ldots, f_{r}\left(\lambda^{2} i h_{r l}\right), f_{r+1}\left(\lambda^{2} i\right), \ldots, f_{m}\left(\lambda^{2} i\right),\right. \\
& \left.0, \ldots, 0, a_{l}\left(\lambda^{2} z_{0}\right) \lambda, 0, \ldots, 0\right) \in \partial G \\
& \operatorname{Im} f_{1}\left(\lambda^{2} i h_{1 l}\right)-\lambda^{2} h_{1 l}\left|a_{l}\left(\lambda^{2} z_{0}\right)\right|^{2} \geq 0 \\
\Rightarrow & \vdots \\
& \operatorname{Im} f_{r}\left(\lambda^{2} i h_{r l}\right)-\lambda^{2} h_{r l}\left|a_{l}\left(\lambda^{2} z_{0}\right)\right|^{2} \geq 0
\end{aligned}
$$

(as well as $\operatorname{Im} f_{r+1}\left(\lambda^{2} i\right)>0, \ldots, \operatorname{Im} f_{m}\left(\lambda^{2} i\right)>0$ ). At least one of these inequalities has to be an equality.

The automorphisms $f_{1}, \ldots, f_{r}$ will now be given specific values. It has been shown in the 4th step that for each $f \in A u t_{u}^{\prime} \tilde{G}=A u t_{u}^{\prime} T\left(\mathbb{R}_{+}^{m}\right)$ there is a torus of dimension $k$ of automorphisms $F \in U$ with $f_{F}=f$. Since $k>0$, there is at least one $F \in U$ with $f_{F}=f$ for every choice of $f \in A u t_{u}^{\prime} \tilde{G}$. Let $f_{2}, \ldots, f_{r}=i d$, and let

$$
f_{1}(z)=\frac{a z+b}{c z+d} \text { with } a:=\frac{2 \operatorname{Re} u_{1}}{\left|u_{1}\right|}, b:=-\left|u_{1}\right|<0, c:=\frac{1}{\left|u_{1}\right|}, \text { and } d:=0
$$

Then $f_{1} \in$ Aut $H^{+}$, since $a, b, c, d \in \mathbb{R}$ with $\operatorname{det}\left(\begin{array}{ll}a & b \\ c & d\end{array}\right)=1$. A trivial calculation shows $f_{1}\left(u_{1}\right)=u_{1}$. Thus $i d \neq f_{1} \in A u t_{u_{1}} H^{+}=A u t_{u_{1}}^{\prime} H^{+}$. Choose $F \in U$ with $f_{F}=f$. It follows from (2) that

$$
\begin{aligned}
& \operatorname{Im} f_{1}\left(\lambda^{2} i h_{1 l}\right)-\lambda^{2} h_{1 l}\left|a_{l}\left(\lambda^{2} z_{0}\right)\right|^{2} \geq 0 \\
& h_{2 l} \lambda^{2}\left(1-\left|a_{l}\left(\lambda^{2} z_{0}\right)\right|^{2}\right) \geq 0 \\
& \vdots \\
& h_{r l} \lambda^{2}\left(1-\left|a_{l}\left(\lambda^{2} z_{0}\right)\right|^{2}\right) \geq 0
\end{aligned}
$$

We will distinguish 2 cases, depending on the behavior of the function $\lambda \mapsto\left|a_{l}\left(\lambda^{2} z_{0}\right)\right|$.

1. Case: $\left|a_{l}\left(\lambda^{2} z_{0}\right)\right| \equiv 1$

In this case we have $\operatorname{Im} f_{1}\left(\lambda^{2} i h_{1 l}\right) \geq \lambda^{2} h_{1 l} \forall \lambda>0$. Since $c \neq 0$, this is in contradiction to part (i) of the following lemma:

Lemma 4.2 Let $u \in H^{+}$, and let $f \in$ Aut $H^{+}$with associated matrix $\left(\begin{array}{ll}a & b \\ c & d\end{array}\right)$, where $c \neq 0$. Then 
(i) there is a $x \in \mathbb{R}^{+}$with $\operatorname{Im} f(i x)<x$.

(ii) if $|d|<1$, there is a $x \in \mathbb{R}^{+}$with $\operatorname{Im} f(i x)>x$.

Proof. A simple calculation shows $\operatorname{Im} f(i x)=\frac{x}{c^{2} x^{2}+d^{2}}$ for all $x \in \mathbb{R}^{+}$. Thus $\operatorname{Im} f(i x)<x \Leftrightarrow c^{2} x^{2}+d^{2}>1 \Leftrightarrow x^{2}>\frac{1-d^{2}}{c^{2}}$. A corresponding result holds if the inequality signs are reversed. This proves both parts of the assertion.

Therefore the first case cannot occur.

2. Case: $\left|a_{l}\left(\lambda^{2} z_{0}\right)\right| \not \equiv 1$

In this case, $\left|a_{l}\left(\lambda^{2} z_{0}\right)\right| \neq 1$ Lebesgue almost everywhere. We will assume from now on that $r>1$, or in other words that at least 2 elements are different from 0 in the $l$-th column of $H$. It follows from (3) that $\left|a_{l}\left(\lambda^{2} z_{0}\right)\right|<1$ Lebesgue almost everywhere. Since equality has to hold in at least one of the inequalities (3), it follows that

$$
\operatorname{Im} f_{1}\left(\lambda^{2} i h_{1 l}\right)=\lambda^{2} h_{1 l}\left|a_{l}\left(\lambda^{2} z_{0}\right)\right|^{2}
$$

for all $\lambda$ outside a set of measure 0 . Consequently,

$$
\operatorname{Im} f_{1}\left(\lambda^{2} i h_{1 l}\right)=\lambda^{2} h_{1 l}\left|a_{l}\left(\lambda^{2} z_{0}\right)\right|^{2} \leq \lambda^{2} h_{1 l}
$$

for all $\lambda \in \mathbb{R}^{+}$. This is in contradiction to part (ii) of the lemma above. In the $l$-th and thus in every column of $H$ there is therefore at most one element different from 0. Since no Siegel domain of the second kind contains an entire complex line, we have that in every column of $H$ there is exactly one element different from 0.

After a suitable permutation of the coordinates, we have

$$
(z, w) \in G \Longleftrightarrow \begin{aligned}
& \operatorname{Im} z_{1}-h_{11}\left|w_{1}\right|^{2}-\cdots-h_{1 l_{1}}\left|w_{l_{1}}\right|^{2}>0 \\
& \operatorname{Im} z_{2}-h_{2, l_{1}+1}\left|w_{l_{1}+1}\right|^{2}-\cdots-h_{2 l_{2}}\left|w_{l_{2}}\right|^{2}>0 \\
& \vdots \\
& \operatorname{Im} z_{m}-h_{m, l_{m-1}+1}\left|w_{l_{m-1}+1}\right|^{2}-\ldots-h_{m l_{m}}\left|w_{l_{m}}\right|^{2}>0
\end{aligned}
$$

with $1 \leq l_{1}<\cdots<l_{m}:=k$.

After the linear change of coordinates

$$
\begin{aligned}
& w_{1}^{\prime}:=\sqrt{h_{11}} w_{1}, \ldots, w_{l_{1}}^{\prime}:=\sqrt{h_{1 l_{1}}} w_{l_{1}}, w_{l_{1}+1}^{\prime}:=\sqrt{h_{2, l_{1}+1}} w_{l_{1}+1}, \ldots, \\
& w_{k}^{\prime}=w_{l_{m}}^{\prime}:=\sqrt{h_{m l_{m}}} w_{l_{m}}
\end{aligned}
$$

$G$ has the form

$$
(z, w) \in G \Longleftrightarrow \begin{aligned}
& \operatorname{Im} z_{1}-\left|w_{1}\right|^{2}-\cdots-\left|w_{l_{1}}\right|^{2}>0 \\
& \operatorname{Im} z_{2}-\left|w_{l_{1}+1}\right|^{2}-\cdots-\left|w_{l_{2}}\right|^{2}>0 \\
& \vdots \\
& \operatorname{Im} z_{m}-\left|w_{l_{m-1}+1}\right|^{2}-\cdots-\left|w_{l_{m}}\right|^{2}>0
\end{aligned}
$$


(we use $w_{j}$ instead of $w_{j}^{\prime}$ for simplicity). It has been shown in the introduction that this domain is biholomorphically equivalent to the product of balls $B_{l_{1}+1} \times B_{l_{2}-l_{1}+1} \times \cdots \times B_{l_{m}-l_{m-1}+1}$. This proves the theorem.

Let $G$ be a domain that fulfills the conditions of theorem 4.1. It will be shown next that the number of factors of the product of balls $G$ is biholomorphically equivalent to can be computed in terms of global geometrical invariants of $G$, as well as by properties of the isotropy groups. This will be used to find characterizations of the two most important special cases of products of balls, namely the ball and the polydisc.

Corollary 4.3 Let $G \in \mathbf{S D 2}_{n}$ be a domain with a point $p \in G$ such that Aut $G$ contains a torus of dimension $n$. Let $(m, k)$ be the type of $G$ and let $C_{p}$ denote the center of $A u t_{p}^{\prime} G$. Then $m=\operatorname{dim} C_{p}$, and $G$ is biholomorphically equivalent to a product of balls of $m$ factors.

Proof. It follows from theorem 4.1 that $G$ is biholomorphically equivalent to a product of balls $B_{k_{1}+1} \times \cdots \times B_{k_{\tilde{m}+1}}$ of $\tilde{m}$ factors for some $\tilde{m} \in \mathbb{N}$. It has been shown in the introduction that the type of this product is $(\tilde{m}, n-\tilde{m})$. Since the type is a biholomorphic invariant, we have $\tilde{m}=m$ as desired.

The isotropy groups of a ball $B_{n}$ are isomorphic to $U(n)$, and the center of $U(n)$ is given by $\left\{e^{i \alpha} \mathbf{I}: \alpha \in \mathbb{R}\right\}$. Consequently, the connected components of the identity of the isotropy groups of $B_{k_{1}+1} \times \cdots \times B_{k_{m}+1}$ are isomorphic to $U\left(k_{1}+1\right) \times \cdots \times U\left(k_{m}+1\right)$, where the center of this group is given by

$$
\left\{\operatorname{diag}(\underbrace{e^{i \alpha_{1}}, \ldots, e^{i \alpha_{1}}}_{k_{1}+1}, \ldots, \underbrace{e^{i \alpha_{m}}, \ldots, e^{i \alpha_{m}}}_{k_{m}+1}): \alpha_{1}, \ldots, \alpha_{m} \in \mathbb{R}\right\}
$$

Thus $\operatorname{dim} C_{p}=m$.

We will use this corollary to give a series of characterizations of the ball and the polydisc.

Corollary 4.4 (A characterization of the ball and the polydisc) A domain $G \in \mathbf{S D 2}{ }_{n}$ is biholomorphically equivalent to a

(i) ball, iff there is a point $p \in G$ such that Aut $t_{p} G$ contains a torus of dimension $n$, and $\operatorname{dim}$ center $A u t_{p}^{\prime} G=1$.

(ii) polydisc, iff there is a point $p \in G$ such that $\operatorname{dim}$ center $A u t_{p}^{\prime} G=n$.

Proof. Part (i) is an immediate consequence of corollary 4.3. Now (ii) will be proved: since $A u t_{p}^{\prime} G$ is compact, the condition $\operatorname{dim}$ center $A u t_{p}^{\prime} G=n$ implies that $A u t_{p}^{\prime} G$ contains a torus of dimension $n$. Now corollary 4.3 shows that $G \subset \mathbb{C}^{n}$ is biholomorphically equivalent to a product of balls of $n$ factors. 
An immediate consequence of part (ii) of the corollary above is the following:

Corollary 4.5 (A characterization of the polydisc) $A$ domain $G \in \mathbf{S D 2}{ }_{n}$ is biholomorphically equivalent to a polydisc, iff there is a point $p \in G$ such that $A u t_{p}^{\prime} G$ is a torus of dimension $n$.

Proof.

Now the type will be used to characterize the ball and the polydisc in a unified way:

Corollary 4.6 (A characterization of the ball and the polydisc) A domain $G \in \mathbf{S D 2}{ }_{n}$ of the type $(m, k)$ is biholomorphically equivalent to a

(i) ball, iff $m=1$, and there is a point $p \in G$ such that Aut $t_{p}$ contains a torus of dimension $n$.

(ii) polydisc, iff $m=n$, and there is a point $p \in G$ such that Aut ${ }_{p} G$ contains a torus of dimension $n$.

Proof.

\section{A counterexample and applications}

Let $G \subset \mathbb{C}^{n}$ be a Reinhardt domain with $0 \in G$. Then $A u t_{0} G$ contains the mappings

$$
\left(z_{1}, \ldots, z_{n}\right) \mapsto\left(e^{i \alpha_{1}} z_{1}, \ldots, e^{i \alpha_{n}} z_{n}\right), \alpha_{1}, \ldots \alpha_{n} \in \mathbb{R}
$$

These mappings obviously form a torus of dimension $n$ in $A u t_{0} G$ (but note that $A u t_{0} G$ is not necessarily a Lie group if $G$ is not bounded). The characterization of theorem 4.1 can therefore be no longer valid in any class of domains that contains a bounded Reinhardt domain $G \ni 0$ that is not biholomorphically equivalent to a product of balls. It will be shown by an example that already the class BNC $\supset$ SD2 of domains biholomorphically equivalent to a bounded domain with a noncompact automorphism group contains such a Reinhardt domain. Thus theorem 4.1 cannot be extended to BNC. As an immediate consequence we get that $\mathbf{B N C}_{n} \backslash \mathbf{S D 2} \mathbf{2}_{n}$ contains topological balls for all $n>1$.

The example is the following well known Thullen domain:

Theorem 5.1 Let $G:=\left\{(z, w) \in \mathbb{C}^{2}:|z|^{2}+|w|^{4}<1\right\}$. Then

(i) the maps $f_{a}(z, w):=\left(\frac{z-a}{1-\bar{a} z}, \frac{w \sqrt[4]{1-|a|^{2}}}{\sqrt{1-\bar{a} z}}\right)$, with $|a|<1$, are automorphisms of $G$,

(ii) $G \in \mathbf{B N C \text { , }}$

(iii) Aut $t_{0} G$ contains a torus of dimension 2 , 
but $G$ is not biholomorphically equivalent to a product of balls (in this case the ball or the polydisc).

Proof. (i) $|z-a|^{2}=|1-\bar{a} z|^{2}-\left(1-|z|^{2}\right)\left(1-|a|^{2}\right)$; therefore

$$
\begin{aligned}
\left|\frac{z-a}{1-\bar{a} z}\right|^{2}+\left|\frac{w \sqrt[4]{1-|a|^{2}}}{\sqrt{1-\bar{a} z}}\right|^{4}= & -\frac{\left(1-|z|^{2}\right)\left(1-|a|^{2}\right)}{|1-\bar{a} z|^{2}}+ \\
& +\frac{|w|^{4}\left(1-|a|^{2}\right)}{|1-\bar{a} z|^{2}} \\
= & 1-\frac{\left(1-|z|^{2}-|w|^{4}\right)\left(1-|a|^{2}\right)}{|1-\bar{a} z|^{2}}<1
\end{aligned}
$$

for $(z, w) \in G$. Thus $f_{a}(G) \subseteq G$. A simple calculation shows that $f_{a} \circ f_{-a}=i d=f_{-a} \circ f_{a}$. Consequently, $f_{a}$ is an automorphism of $G$.

(ii) If Aut $G$ would be compact, the orbit of $(0,0)$ under Aut $G$ would be compact as well. But the image of $(0,0)$ under the mappings $f_{a}$ is exactly $\{(a, 0):|a|<1\}$, which is a contradiction. Thus Aut $G$ is noncompact.

(iii) Since $G$ is a bounded Reinhardt domain that contains the origin, the discussion above shows that $A u t_{0} G$ contains a torus of dimension 2 .

To prove that $G$ is not biholomorphically equivalent to the ball or the polydisc, we need the following theorem of T. Sunada ([Sun78]):

Theorem 5.2 (Sunada) Two bounded Reinhardt domains $G_{1}, G_{2}$ in $\mathbb{C}^{n}$ are biholomorphically equivalent, iff there is a linear map $\phi: \mathbb{C}^{n} \rightarrow \mathbb{C}^{n}$ of the form $z_{j} \mapsto r_{j} z_{\sigma(j)}$ for all $j, r_{j} \in \mathbb{R}^{+}, \sigma$ a permutation of $(1, \ldots, n)$, with $\phi\left(G_{1}\right)=G_{2}$.

Obviously, $G$ is not equivalent to the ball or the polydisc under a linear map of this form. This completes the proof.

Corollary 5.3 SD2 $\varsubsetneqq$ BNC. Furthermore, BNC $_{n} \backslash \mathbf{S D 2}_{n}$ contains topological balls for all $n>1$.

Remark Since the only bounded simply connected domain in $\mathbb{C}^{1}$ up to biholomorphism is the unit disc, $\mathbf{B N C}_{1} \backslash \mathbf{S D 2} 2_{1}$ can of course not contain a (topological) disc.

Proof. (of corollary 5.3.) Let $G:=\{(z, w): \operatorname{Im} z-H(w, w) \in C\}$ be a Siegel domain of the second kind. Then $(z, w) \mapsto(\lambda z, \sqrt{\lambda} w)$ is an automorphism of $G$ for all $\lambda \in \mathbb{R}^{+}$. Let $\left(z_{0}, w_{0}\right)$ be in $G$. If Aut $G$ would be compact, then the orbit $A u t G \cdot\left(z_{0}, w_{0}\right)$ would be compact as well. But this is in contradiction to $\left\{\left(\lambda z_{0}, \sqrt{\lambda} w_{0}\right): \lambda \in \mathbb{R}^{+}\right\} \subset$ Aut $G \cdot\left(z_{0}, w_{0}\right)$. Thus $A u t G$ is noncompact. 
An example of a non simply connected bounded domain $G$ in $\mathbb{C}^{1}$ with noncompact automorphism group can be found in [Kra83]. Thus $\mathbf{S D 2}_{1} \neq$ $\mathbf{B N C}_{1}$. Now let $n \geq 2$, and let $G:=\left\{(z, w) \in \mathbb{C}^{2}:|z|^{2}+|w|^{4}<\right.$ $1\} \times B_{n-2}$. It follows from theorem 5.1 and theorem 4.1 that $G \notin \mathbf{S D 2}$; nevertheless, Aut $G$ is noncompact. And since $G$ is a complete Reinhardt domain that contains the origin, $G$ is a topological ball. Thus $\mathbf{B N C}_{n} \backslash \mathbf{S D 2}{ }_{n}$ contains topological balls for all $n>1$.

It follows immediately from the discussion at the beginning of this section and theorem 4.1 that a bounded Reinhardt domain that contains the origin is biholomorphically equivalent to a Siegel domain of the second kind, iff it is biholomorphically equivalent to a product of balls. Thus we have computed the intersection of SD2 with the class of all domains biholomorphically equivalent to a bounded Reinhardt domain that contains the origin. This result will now be extended to the class $R D$ of all domains biholomorphically equivalent to any Reinhardt domain. Let $\mathbf{P B}$ denote the class of all domains biholomorphically equivalent to a product of balls.

Theorem 5.4 SD2 $\cap \mathrm{RD}=\mathrm{PB}$

Proof. Let $G \subset \mathbb{C}^{n}$ be a Reinhardt domain with $0 \notin G$. It will be shown that $G$ cannot be biholomorphically equivalent to a Siegel domain of the second kind. We will use the fact that Siegel domains of the second kind are convex.

1. Case: Assume that for all $j$ with $1 \leq j \leq n$ there is a point $z=$ $\left(z_{1}, \ldots, z_{n}\right) \in G$ with $z_{j}=0$.

It is well known that in this case every holomorphic function on $G$ can be extended to the complete hull

$$
\tilde{G}:=\left\{\left(r_{1} z_{1}, r_{2} z_{2}, \ldots, r_{n} z_{n}\right):\left(z_{1}, \ldots, z_{n}\right) \in G, 0 \leq r_{j} \leq 1\right\}
$$

of $G$. Since $0 \notin G$ we have $\tilde{G} \supsetneqq G$, and $G$ is not a domain of holomorphy. Thus it cannot be biholomorphically equivalent to a convex domain.

2. Case: Assume that there is a $j$ such that $z_{j} \neq 0$ for all $z \in G$.

Let $p r_{j}$ denote the projection of $\mathbb{C}^{n}$ onto the $j$-th coordinate. Let $z=$ $\left(z_{1}, \ldots, z_{n}\right)$ be in $G$, and let $\gamma:[0,1] \rightarrow \mathbb{C}^{n}, t \mapsto\left(e^{2 \pi i t} z_{1}, \ldots, e^{2 \pi i t} z_{n}\right)$. Since $G$ is a Reinhardt domain, we have that $\gamma \subset G$. The projection $p r_{j}(\gamma)$ of this curve is a circle around the origin. Since $0 \notin p r_{j}(G),\left[p r_{j}(\gamma)\right] \in$ $\pi_{1}\left(p r_{j}(G)\right)$ is nontrivial. Therefore $[\gamma] \in \pi_{1}(G)$ is nontrivial as well, and $G$ cannot be biholomorphically equivalent to a convex domain.

Let now $G$ be a Reinhardt domain that is biholomorphically equivalent to a Siegel domain of the second kind. It has been shown above that $0 \in G$. Therefore the $n$-dimensional torus of linear mappings

$$
\left(z_{1}, \ldots, z_{n}\right) \mapsto\left(e^{i \alpha_{1}} z_{1}, \ldots, e^{i \alpha_{n}} z_{n}\right), \alpha_{1}, \ldots \alpha_{n} \in \mathbb{R}
$$


is in $A u t_{0} G$. It now follows from theorem 4.1 that $G$ is biholomorphically equivalent to a product of balls.

Using the theorem of Sunada, we can now give a complete description of all Reinhardt domains that are biholomorphically equivalent to a Siegel domain of the second kind.

Corollary 5.5 A Reinhardt domain $G$ in $\mathbb{C}^{n}$ is biholomorphically equivalent to a Siegel domain of the second kind, iff it is a product of ellipsoids of the form

$$
G=\left\{\begin{array}{c}
\left|\frac{z_{\sigma(1)}}{s_{1}}\right|^{2}+\quad \cdots+\left|\frac{z_{\sigma\left(n_{1}\right)}}{s_{n_{1}}}\right|^{2}<1 \\
z \in \mathbb{C}^{n}:\left.\quad \frac{z_{\sigma\left(n_{1}+\cdots+n_{k-1}+1\right)}}{s_{n_{1}+\cdots+n_{k-1}+1}}\right|^{2}+\cdots+\left|\frac{z_{\sigma\left(n_{1}+\cdots+n_{k}\right)}}{s_{n_{1}+\cdots+n_{k}}}\right|^{2}<1
\end{array}\right\}
$$

where $n_{1}+\cdots+n_{k}=n, s_{1}, \ldots, s_{n} \in \mathbb{R}^{+}$, and $\sigma$ a permutation of $(1, \ldots, n)$.

Proof. This follows immediately from theorem 5.4 and theorem 5.2 of Sunada.

Finally, theorem 5.4 can be reformulated to give another characterization of products of balls.

Corollary 5.6 A domain is biholomorphically equivalent to a product of balls, iff it is biholomorphically equivalent to a Siegel domain of the second kind and to a Reinhardt domain.

Proof.

\section{References}

[Hel78] S. Helgason, Differential geometry, Lie groups, and symmetric spaces, Academic Press, New York, 1978

[KMO70] W. Kaup, Y. Matsushima, T. Ochiai, On the automorphisms and equivalences of generalized Siegel domains, Amer. J. Math, 92 (1970), 475-497

[Koe62] M. Koecher, Jordan algebras and their applications, Lecture Notes, University of Minnesota, 1962

[Kra83] S. Krantz, Automorphisms, Complex Analysis II, (C. Berenstein, ed.), Lecture Notes in Mathematics, vol. 286, Springer, New York, 1983, pp. 10-97

[Mur72] S. Murakami, On automorphisms of Siegel domains, Lecture Notes in Mathematics, vol. 286, Springer, New York, 1972 
[Nar71] R. Narasimhan, Several complex variables, Chicago Lectures in Mathematics, The University of Chicago, 1971

[Rot71] O. S. Rothaus, Automorphisms of Siegel domains, Trans. Amer. Math. Soc. 162 (1971), 351-382

[Sun78] T. Sunada, Holomorphic equivalence problems for bounded Reinhardt domains, Math. Ann. 235 (1978), 111-128

[Won77] B. Wong, Characterizations of the ball in $\mathbb{C}^{n}$ by its automorphism group, Invent. Math. 41 (1977), 253-257 